\title{
32. HISTORY OF ICE RAFTING AT LEG 114 SITES, SUBANTARCTIC/SOUTH ATLANTIC1
}

\author{
Carl P. Allen ${ }^{2}$ and Detlef A. Warnke ${ }^{2}$
}

\begin{abstract}
All holes drilled during Leg 114 contained ice-rafted debris. Analysis of samples from Hole 699A, Site 701, and Hole 704A yielded a nearly complete history of ice-rafting episodes.

The first influx of ice-rafted debris at Site 699 , on the northeastern slope of the Northeast Georgia Rise, occurred at a depth of $69.94 \mathrm{~m}$ below seafloor (mbsf) in sediments of early Miocene age ( $23.54 \mathrm{Ma})$. This material is of the same type as later ice-rafted debris, but represents only a small percentage of the coarse fraction. Significant ice-rafting episodes occurred during Chron 5.

Minor amounts of ice-rafted debris first reached Site 701, on the western flank of the Mid-Atlantic Ridge (8.78 $\mathrm{Ma}$ at $200.92 \mathrm{mbsf}$ ), and more arrived in the late Miocene (5.88 Ma). The first significant quantity of sand and gravel appeared at a depth of $107.76 \mathrm{mbsf}(4.42 \mathrm{Ma})$. Site 704 , on the southern part of the Meteor Rise, received very little or no ice-rafted debris prior to $2.46 \mathrm{Ma}$. At this time, however, the greatest influx of ice-rafted debris occurred at this site. This time of maximum ice rafting correlates reasonably well with influxes of ice-rafted debris at Sites 701 $(2.24 \mathrm{Ma})$ and $699(2.38 \mathrm{Ma})$, in consideration of sample spacing at these two sites. These peaks of ice rafting may be Sirius till equivalents, if the proposed Pliocene age of Sirius tills can be confirmed. After about 1.67 Ma, the apparent mass-accumulation rate of the sediments at Site 704 declined, but with major fluctuations. This decline may be the result of a decrease in the rate of delivery of detritus from Antarctica due to reduced erosive power of the glaciers or a northward shift in the Polar Front Zone, a change in the path taken by the icebergs, or any combination of these factors.
\end{abstract}

\section{INTRODUCTION}

Antarctica is surrounded by a halo of glacial-marine sediments that contain a record of the glaciation and glacial history of that continent (Warnke, 1970). In the area of investigation along the Polar Front Zone, glacial-marine sedimentation is dominated by ice rafting (Anderson et al., 1983). Ice-rafted debris has a widespread distribution, but deposits of this type are typically overlooked in discussions of sedimentary environments and facies. Our limited knowledge of ice-rafted sediments is due to their restricted accessibility. The collection of Southern Ocean cores by drill ship has greatly increased our capabilities to investigate such environments. The sites studied for this investigation are Sites 699, 701, and 704 of Ocean Drilling Program (ODP) Leg 114.

\section{Location}

Site 699 is in the western region of the East Georgia Basin $\left(51^{\circ} 32.531^{\prime} \mathrm{S}, 30^{\circ} 40.603^{\prime} \mathrm{W}\right)$ on the northeastern slope of the Northeast Georgia Rise in a water depth of 3725 m (Fig. 1). The site consists of a single hole, and has a sedimentary sequence that is almost entirely pelagic and mostly biogenic.

Site 701 is on the western flank of the Mid-Atlantic Ridge $\left(51^{\circ} 59.07^{\prime} \mathrm{S}, 23^{\circ} 2.73^{\prime} \mathrm{W}\right.$ ) in a water depth of $4634 \mathrm{~m}$ (Fig. 1), about $60 \mathrm{~km}$ east of the Islas Orcadas Rise in an oceanic gateway permitting deep circulation between the South Atlantic and the Weddell Basin. Site 701 is the only Leg 114 site under the influence of Antarctic Bottom Water, and its sedimentary record is significantly different from the other sites under the influence of Circumpolar Deep Water. The strati-

\footnotetext{
${ }^{1}$ Ciesielski, P. F., Kristoffersen, Y., et al., 1991. Proc. ODP, Sci. Results, 114: College Station, TX (Ocean Drilling Program).

2 Department of Geological Sciences, California State University, Hayward, Hayward, CA 94542 (Present address: Engineering-Science, Inc., 600 Bancroft Way, Berkeley, CA 94710).
}

graphic section consists mostly of biosiliceous and diatom ooze, siliceous clay/mud, and clay-bearing diatom ooze.

Site 704 is on the southern part of the Meteor Rise $\left(46^{\circ} 52.757^{\prime} \mathrm{S}, 7^{\circ} 25.250^{\prime} \mathrm{E}\right)$ in a water depth of $2531 \mathrm{~m}$ (Fig. 1). The site is on a plain west of and deeper than the basement crest of the Meteor Rise, an aseismic ridge formed by extensive $\mathrm{Pa}$ leocene-Eocene volcanism at an extension of the Mid-Atlantic Ridge. The sedimentary sequence consists primarily of calcareous oozes and chalks and of siliceous oozes with little influx of terrigenous sediments by currents or ice rafting.

\section{Purpose of Investigation}

The principal objectives of this report are to

1. describe the fluctuations in concentration and apparent mass-accumulation rate (AMAR) of ice-rafted debris from the Miocene to the Quaternary in sediments obtained during Leg 114 , and

2. attempt to relate these observations to other records of subantarctic ice-rafted debris accumulation rates and the late Cenozoic glacial history of the region.

The area is ideally suited for the study of ice-rafted debris because it is in the vicinity of the present-day Polar Front Zone, where a large portion of iceberg melting occurs, and contains only the ice-rafted or distal facies of the glacialmarine facies association as defined by Molnia (1983). The sites are also located on flat or gently sloping oceanic crust, as opposed to a basinal environment. The sites, therefore, reveal the development of ice rafting in this area, little disturbed by mass wasting.

\section{METHODS}

Twenty plugs of approximately $1 \mathrm{~cm}^{3}$ were taken about every $50 \mathrm{~cm}$ at Sites 699 and 701 and about every $150 \mathrm{~cm}$ at Site 704. All samples were freeze-dried aboard ship or kept frozen until the shorebased procedure could be performed (see Allen, 1989, for a detailed description of the techniques). Sample volume and weight were determined so that the 


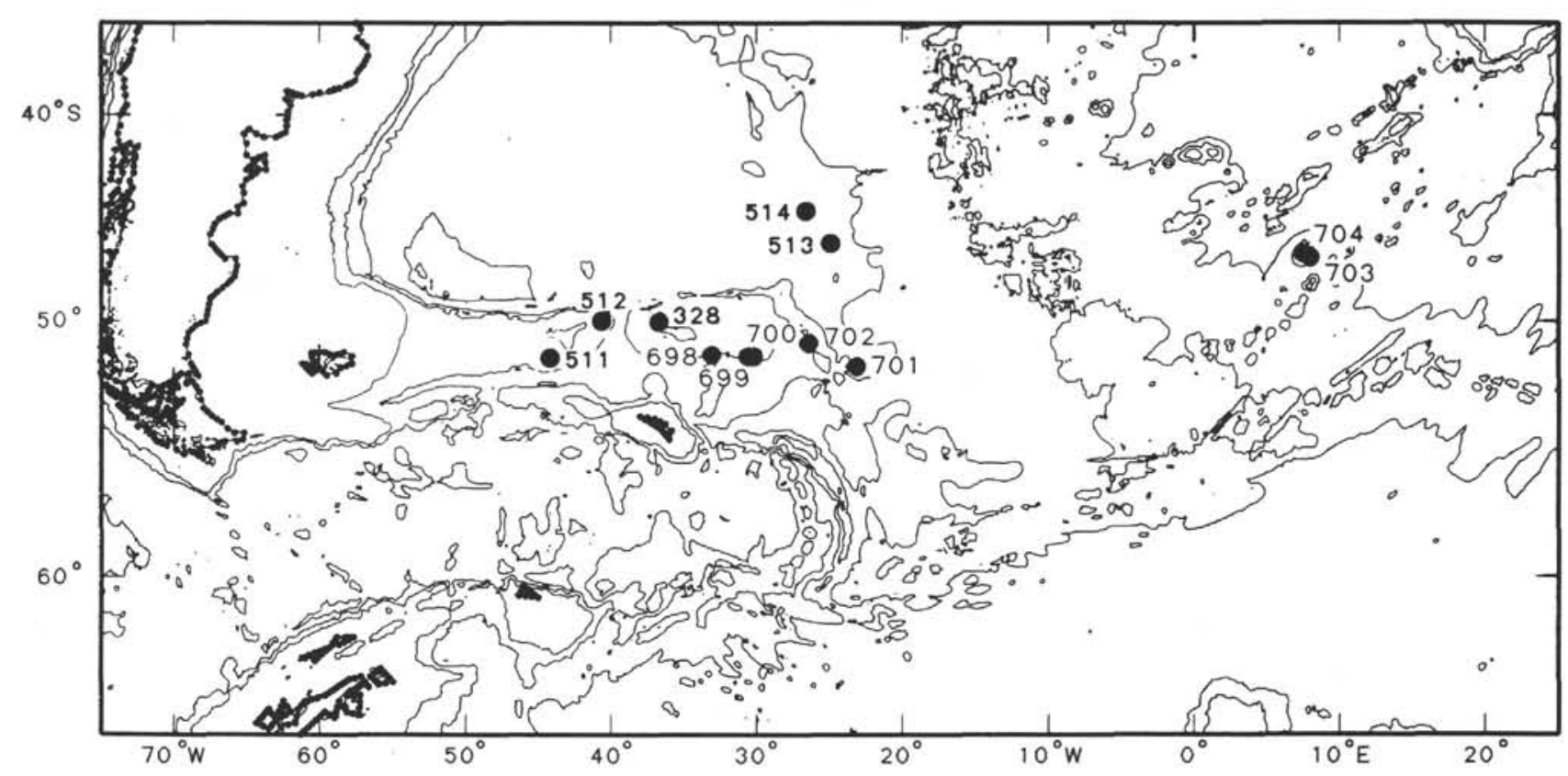

Figure 1. Leg 114 Sites 698-704 in the South Atlantic sector of the Southern Ocean.

dry-bulk density could be calculated. Each sample was disaggregated in the dispersing agent and washed through a $63-\mu \mathrm{m}$ sieve. The sand fraction was processed using a combination of methods described by Molnia (1983) and Bornhold (1983). Using $250 \mu \mathrm{m}$ as a lower limit (Bornhold, 1983) instead of 500 $\mu \mathrm{m}$ (Molnia, 1983) facilitates comparing these results with Bornhold's results for the Southern Ocean.

After the different size fractions were weighed, the 250 $\mu \mathrm{m}$ - to $2-\mathrm{mm}$-sized fraction was examined under a binocular microscope. The ratio of biogenic to terrigenous material was determined by grain counts of representative subsamples to determine the volume percent of ice-rafted debris in this fraction. The volume percent of ice-rafted debris multiplied by the weight of the $250-\mu \mathrm{m}$ to $2-\mathrm{mm}$ fraction results in the minimum weight of ice-rafted debris in the sample. The ratio of the minimum weight of the ice-rafted debris to total sample weight results in a concentration of ice-rafted debris in $\mathrm{mg} / \mathrm{g}$ (Tables 1-3). The ratio of the total bulk weight to total volume yields the dry-bulk density. The stratigraphic framework shown in Tables 4 through 6 was used to determine sedimentation rates, which when multiplied by the dry-bulk density yield a mass-accumulation rate (MAR). The MAR multiplied by percent ice-rafted debris results in the final "ice-rafted debris apparent mass-accumulation rate" (AMAR) expressed as $\mathrm{mg} / \mathrm{cm}^{2} / 10^{3} \mathrm{yr}$.

\section{RESULTS}

\section{Site 699}

The light brownish gray, pale olive, and gray diatom ooze of Core 114-699A-1H shows two periods of increased influx of ice-rafted debris near the top, at $0.70 \mathrm{mbsf}(0.220 \mathrm{Ma})$ and 7.41 mbsf ( $0.57 \mathrm{Ma}$ ) (Fig. 2). The much greater AMARs at Site 699 relative to those of Sites 701 and 704 (compare Figs. 2, 3, and 4) may well be an artifact. One possible reason is the winnowing of the fine biogenic material as well as the fine ice-rafted debris during settling in the vicinity of Site 699 . This would have produced an "artificially" high percentage of the coarse ice-rafted debris examined in this study, which resulted in the much higher AMARs.
The few samples from the greenish gray diatom ooze and volcanic ash-bearing to volcanic ash diatom clay of Core 114-699A-2H are widely spaced, but show a significant influx of ice-rafted debris at a depth of $14.40 \mathrm{mbsf}$ (1.36 Ma) (Fig. 2).

The greenish gray diatom-bearing volcanic ash clay of Core 114-699A-4H contains a peak of ice-rafted debris at 28.22 mbsf (2.38 Ma) (Fig. 2). Farther below, ice-rafted debris AMARs are relatively high at $31.04 \mathrm{mbsf}(2.58 \mathrm{Ma})$, and then decrease abruptly downward. The greenish gray volcanic ash-bearing diatom clay and volcanic ash diatom ooze of Core 114-699A-5H appear to have a hiatus at or near $45.27 \mathrm{mbsf}$, cutting out the Gilbert Chron between 4.20 and $4.70 \mathrm{Ma}$. The samples examined from the light olive gray volcanic ashbearing clayey diatom ooze and volcanic ash-bearing diatom ooze of Core 114-699A-6H of latest Miocene to early Gilbert Chron age show sawtoothlike fluctuations produced by major episodes in the waxing and waning influx of ice-rafted debris.

According to P. F. Ciesielski (pers. comm., 1990), several hiatuses are identified within the Miocene of Hole 699A. The first separates the upper reversed polarity interval of Chron C3AR from lower Chron C5N (about 6.0-10.0 Ma) and occurs between Samples 114-699A-7H-CC and 114-699A-8H-1, 40 $\mathrm{cm}(66.02-63.28 \mathrm{mbsf})$. The lower upper Miocene sediment of Section 114-699A-8H-1 is in turn separated by another hiatus (60.02-66.15 mbsf) from middle Miocene sediment in Section 114-699A-8H-2, which probably represents the lower Nitzschia denticuloides diatom Zone. Yet another hiatus occurs between Sections 114-699A-8H-2 and 114-699A-8H-3, separating the middle Miocene from the lowermost lower Miocene. The depth of this hiatus is critical in determining the age of the oldest ice-rafted debris in the hole. This hiatus occurs between 69.20 and $67.52 \mathrm{mbsf}$; therefore, the oldest ice-rafted debris at 69.94 mbsf is a minimum of $74 \mathrm{~cm}$ below the hiatus and within Chron $\mathrm{C} 6 \mathrm{CN}$. This ice-rafted debris has an age of about $23.54 \mathrm{Ma}$.

\section{Site 701}

Core $114-701 \mathrm{~A}-2 \mathrm{H}$ is a greenish gray to gray mud- to ash-bearing diatom ooze with an erratic pattern. Peaks indicate increased ice rafting at depths of $12.50 \mathrm{mbsf}(0.54 \mathrm{Ma})$, 
Table 1. Amount of coarse-grained ice-rafted debris, Hole 699A.

\begin{tabular}{|c|c|c|c|}
\hline $\begin{array}{l}\text { Sample depth } \\
\text { (mbsf) }\end{array}$ & $\begin{array}{l}\text { Coarse-grained } \\
\text { ice-rafted debris } \\
(\mathrm{mg} / \mathrm{g})\end{array}$ & $\begin{array}{l}\text { Sample depth } \\
\text { (mbsf) }\end{array}$ & $\begin{array}{l}\text { Coarse-grained } \\
\text { ice-rafted debris } \\
(\mathrm{mg} / \mathrm{g})\end{array}$ \\
\hline 0.20 & 5.71 & 38.76 & 6.51 \\
\hline 0.70 & 64.98 & 39.43 & 5.59 \\
\hline 1.42 & 9.44 & 40.08 & 4.94 \\
\hline 1.70 & 9.12 & 40.28 & 13.00 \\
\hline 2.20 & 1.02 & 40.93 & 27.34 \\
\hline 2.92 & 10.76 & 41.32 & 16.09 \\
\hline 3.20 & 14.55 & 41.72 & 33.96 \\
\hline 3.70 & 19.03 & 42.39 & 12.20 \\
\hline 4.42 & 1.39 & 42.82 & 2.90 \\
\hline 4.70 & 1.74 & 43.43 & 34.99 \\
\hline 5.19 & 0.21 & 43.88 & 28.68 \\
\hline 5.92 & 3.36 & 47.10 & 11.62 \\
\hline 6.19 & 3.15 & 47.70 & 51.04 \\
\hline 6.59 & 14.30 & 48.30 & 10.48 \\
\hline 7.41 & 60.95 & 49.06 & 27.23 \\
\hline 7.70 & 5.32 & 49.33 & 29.36 \\
\hline 8.19 & 26.71 & 49.80 & 5.67 \\
\hline 9.90 & 23.37 & 50.61 & 10.79 \\
\hline 11.40 & 51.56 & 51.01 & 17.68 \\
\hline 12.90 & 6.34 & 51.43 & 16.79 \\
\hline 14.40 & 15.68 & 51.89 & 2.66 \\
\hline 18.63 & 22.71 & 52.17 & 4.70 \\
\hline 18.87 & 51.00 & 52.80 & 17.20 \\
\hline 19.46 & 23.44 & 53.39 & 3.32 \\
\hline 19.91 & 12.00 & 53.57 & 28.22 \\
\hline 20.55 & 12.33 & 54.43 & 24.58 \\
\hline 21.05 & 16.22 & 56.33 & 12.48 \\
\hline 21.41 & 55.20 & 56.65 & 9.83 \\
\hline 21.86 & 37.21 & 57.41 & 13.70 \\
\hline 22.48 & 28.50 & 57.92 & 25.40 \\
\hline 22.80 & 58.67 & 58.29 & 10.29 \\
\hline 23.34 & 13.18 & 58.80 & 5.14 \\
\hline 23.72 & 22.42 & 59.30 & 8.03 \\
\hline 27.69 & 17.89 & 60.17 & 15.53 \\
\hline 28.22 & 21.35 & 60.51 & 21.56 \\
\hline 28.74 & 17.26 & 60.78 & 11.60 \\
\hline 29.32 & 11.30 & 61.20 & 10.90 \\
\hline 29.98 & 8.36 & 62.06 & 5.00 \\
\hline 30.40 & 16.00 & 62.25 & 3.20 \\
\hline 30.67 & 58.55 & 62.37 & 0.20 \\
\hline 31.04 & 45.31 & 66.51 & 0.12 \\
\hline 31.90 & 34.50 & 66.96 & 1.79 \\
\hline 32.14 & 6.39 & 67.37 & 0.00 \\
\hline 33.95 & 12.81 & 67.96 & 0.03 \\
\hline 34.30 & 18.67 & 68.43 & 0.00 \\
\hline 35.04 & 12.25 & 68.85 & 0.00 \\
\hline 37.42 & 12.00 & 69.49 & 0.00 \\
\hline 37.83 & 8.27 & 69.94 & 0.02 \\
\hline 38.54 & 16.35 & & \\
\hline
\end{tabular}

16.05 mbsf (0.69 Ma), and $17.55 \mathrm{mbsf}(0.74 \mathrm{Ma}$ ) (Fig. 3). Core $114-701 \mathrm{~A}-3 \mathrm{H}$ shows a major amount near the bottom, at a depth of 26.50 mbsf $(0.85 \mathrm{Ma})$.

Examination of the olive gray to gray mud- and ash-bearing diatom ooze of Core $114-701 \mathrm{~A}-5 \mathrm{H}$ revealed that a period of significant influx of ice-rafted debris occurred at a depth of 41.12 mbsf (1.56 Ma). A group of samples from the bottom portion of the core, between $44.02 \mathrm{mbsf}(1.64 \mathrm{Ma})$ and $45.56 \mathrm{mbsf}(1.68$ $\mathrm{Ma}$ ), shows significant ice-rafted debris accumulation rates.

Two major influxes of ice-rafted debris occur in the diatom ooze of Core 114-701A-6H at $53.12 \mathrm{mbsf}(1.95 \mathrm{Ma})$ and 55.16 mbsf (2.02 Ma). A significant peak of ice-rafted debris occurs at 61.88 mbsf (2.24 Ma) (Fig. 3). The Gauss sediment at the bottom of Hole 701A overlaps the top of Hole 701B (the upper $4 \mathrm{~m}$ ) above a Gauss hiatus (3.11-2.63 Ma). Just below this hiatus is a cluster of ice-rafted debris peaks signifying smaller ice-rafting episodes.

A significant influx of ice-rafted debris was identified in the greenish gray to gray ash- and mud-bearing diatom ooze of Core $114-701 \mathrm{~B}-2 \mathrm{H}$ at a depth of $81.90 \mathrm{mbsf}(3.61 \mathrm{Ma})$, and a minor peak occurs in Core 114-701B-3H at 89.27 mbsf (3.82 Ma). The first major influx of ice-rafted debris at Hole 701B occurs in the greenish gray to light olive gray ash- and mud-bearing diatom ooze of Core 114-701B-4H at a depth of $107.76 \mathrm{mbsf}$ (4.42 Ma). The first significant influx of ice-rafted debris at Hole 701B occurs at a depth of $143.94 \mathrm{mbsf}$ (5.88 Ma).

Very little ice-rafted debris was observed in the samples below this depth. Between Cores 114-701B-12X and 114$701 \mathrm{~B}-13 \mathrm{X}$ is apparently a hiatus of at least $1.05 \mathrm{~m} . \mathrm{y}$. (about 6.55-7.60 Ma), according to micropaleontological and paleomagnetic evidence from Hole 701C, where the hiatus is better dated (P. F. Ciesielski, pers. comm., 1990). The deepest sample examined in Hole 701B is from a depth of $200.92 \mathrm{mbsf}$ $(8.78 \mathrm{Ma})$ and has a little ice-rafted debris (Fig. 3).

\section{Site 704}

The youngest sample from Hole 704A is from a depth of 2.22 mbsf (isotopic stage 5; Gard and Crux, this volume) and contains no ice-rafted debris. The remainder of the pale yellow to white calcareous-bearing diatom ooze, calcareous ooze, and nannofossil ooze of Core 114-704A-1H produces a very minor influx at a depth of 3.72 mbsf (isotopic stage 7; Gard and Crux, this volume). The white to light gray diatom ooze, calcareous-bearing diatom ooze, and diatomaceous calcareous ooze of Core 114-704A-3H show a significant influx of ice-rafted debris at a depth of 22.18 mbsf (between 620,000 and 730,000 yr B.P.; P. F. Ciesielski, pers. comm., 1990). Core $114-704 \mathrm{~A}-6 \mathrm{H}$ is a white to light olive gray calcareous ooze, diatomaceous calcareous ooze, calcareous diatom ooze, and diatom ooze that show a significant influx of ice-rafted debris at a depth of $47.40 \mathrm{mbsf}(1.03 \mathrm{Ma})$. The white, light gray, and pale olive calcareous diatom ooze of Core 114-704A$9 \mathrm{H}$ contains a minor influx of ice-rafted debris at a depth of 80.41 mbsf (1.53 Ma).

Significant influxes of ice-rafted debris are identified in the light gray to white calcareous diatom ooze of Core 114-704A$11 \mathrm{H}$ at a depth of $101.82 \mathrm{mbsf}(1.79 \mathrm{Ma})$ and in Core $114-704 \mathrm{~A}-12 \mathrm{H}$ at depths of $105.92 \mathrm{mbsf}(1.83 \mathrm{Ma})$ and 107.42 mbsf $(1.85 \mathrm{Ma})$. The white to light gray siliceous calcareous ooze and calcareous siliceous ooze of Core $114-704 \mathrm{~A}-14 \mathrm{H}$ show a cyclic alternation of minor amounts of ice-rafted debris and no ice-rafted debris of about every $26,000 \mathrm{yr}$.

A large influx of ice-rafted debris was noted in the light gray siliceous-bearing to siliceous calcareous ooze at the top of Core 114-704A-17X at a depth of $150.42 \mathrm{mbsf}(2.29 \mathrm{Ma})$. The white to light gray siliceous calcareous ooze to calcareous siliceous ooze of Core 114-704A-18X has the most prominent influx of ice-rafted debris in Hole 704A at a depth of 167.42 mbsf (2.46 Ma), which has an AMAR of $38.04 \mathrm{mg} / \mathrm{cm}^{2} / 10^{3} \mathrm{yr}$ (Fig. 4).

Very little or no ice-rafted debris is in the remainder of Hole 704A except for minor influxes at depths of $173.92 \mathrm{mbsf}$ (2.79 Ma), $176.92 \mathrm{mbsf}(2.95 \mathrm{Ma})$, and $185.12 \mathrm{mbsf}$ (3.33 Ma). The earliest minor influx of ice-rafted debris in Hole 704A is at the top of Core 114-704A-23X at a depth of $208.00 \mathrm{mbsf}$ (4.19 Ma).

\section{DISCUSSION}

\section{Miocene}

The first occurrence of a few sand-sized quartz grains of ice-rafted debris at Site 699 was at about $23.5 \mathrm{Ma}(69.94 \mathrm{mbsf})$, followed by another rare occurrence at $10.7 \mathrm{Ma}$. These few isolated grains may have been transported by seasonal pack ice, which then rafted the grains into the study area (Plafker et al., 1977), or they may have been primarily transported by small bergs from outlet glaciers and small ice shelves in East Antarctica (Ciesielski et al., 1982). 
Table 2. Amount of coarse-grained ice-rafted debris, Site 701.

\begin{tabular}{|c|c|c|c|c|c|}
\hline $\begin{array}{l}\text { Sample depth } \\
\text { (mbsf) }\end{array}$ & $\begin{array}{l}\text { Coarse-grained } \\
\text { ice-rafted debris } \\
\qquad(\mathrm{mg} / \mathrm{g})\end{array}$ & $\begin{array}{l}\text { Sample depth } \\
\text { (mbsf) }\end{array}$ & $\begin{array}{l}\text { Coarse-grained } \\
\text { ice-rafted debris } \\
(\mathrm{mg} / \mathrm{g})\end{array}$ & $\begin{array}{l}\text { Sample depth } \\
\text { (mbsf) }\end{array}$ & $\begin{array}{l}\text { Coarse-grained } \\
\text { ice-rafted debris } \\
\qquad(\mathrm{mg} / \mathrm{g})\end{array}$ \\
\hline \multicolumn{6}{|l|}{ Hole 701A } \\
\hline 5.38 & 1.61 & 53.12 & 13.09 & 101.76 & 0.53 \\
\hline 5.92 & 0.00 & 53.38 & 5.48 & 102.42 & 0.48 \\
\hline 6.49 & 0.80 & 54.00 & 1.68 & 102.97 & 0.22 \\
\hline 7.07 & 2.74 & 54.62 & 2.45 & 103.26 & 1.69 \\
\hline 7.53 & 1.22 & 55.16 & 9.51 & 103.92 & 0.53 \\
\hline 8.02 & 0.64 & 55.47 & 8.60 & 104.47 & 0.60 \\
\hline 8.55 & 1.78 & 55.78 & 2.47 & 104.76 & 0.42 \\
\hline 8.95 & 2.53 & 56.37 & 4.24 & 105.42 & 1.18 \\
\hline 9.50 & 1.38 & 56.78 & 3.84 & 106.26 & 0.50 \\
\hline 10.05 & 0.94 & 57.22 & 0.55 & 106.92 & 1.39 \\
\hline 10.50 & 0.12 & 57.87 & 6.09 & 107.47 & 2.08 \\
\hline 11.00 & 0.25 & 58.28 & 4.00 & 107.76 & 8.14 \\
\hline 11.55 & 0.59 & 58.72 & 1.79 & 108.82 & 0.29 \\
\hline 12.00 & 1.91 & 59.16 & 1.01 & 110.32 & 1.06 \\
\hline 12.50 & 5.50 & 59.62 & 1.46 & 111.82 & 0.36 \\
\hline 13.05 & 4.76 & 60.02 & 0.34 & 113.32 & 0.84 \\
\hline 13.50 & 0.20 & 60.52 & 3.62 & 114.82 & 2.71 \\
\hline 14.03 & 0.65 & 61.02 & 0.62 & 116.32 & 1.76 \\
\hline 14.55 & 2.82 & 61.72 & 2.53 & 118.31 & 3.34 \\
\hline 15.00 & 1.22 & 61.88 & 5.73 & 119.40 & 0.76 \\
\hline 15.50 & 1.58 & 66.73 & 3.83 & 120.90 & 1.44 \\
\hline 16.05 & 5.62 & 68.23 & 2.67 & 123.27 & 0.33 \\
\hline 16.50 & 0.34 & 69.73 & 2.13 & 123.76 & 0.10 \\
\hline 17.00 & 3.77 & 71.23 & 3.47 & 127.68 & 1.05 \\
\hline 17.55 & 5.23 & & & 129.19 & 0.49 \\
\hline 19.00 & 0.58 & Hole $701 \mathrm{~B}$ & & 130.69 & 0.14 \\
\hline 19.54 & 0.51 & 71.87 & 0.52 & 132.12 & 0.56 \\
\hline 20.10 & 0.12 & 72.27 & 0.25 & 133.68 & 0.74 \\
\hline 20.50 & 0.02 & 72.82 & 4.08 & 134.66 & 0.80 \\
\hline 21.04 & 0.13 & 73.37 & 4.66 & 137.10 & 0.73 \\
\hline 21.60 & 0.10 & 73.77 & 0.83 & 137.44 & 0.19 \\
\hline 22.00 & 0.20 & 74.32 & 0.49 & 137.72 & 0.31 \\
\hline 22.54 & 0.12 & 74.87 & 1.79 & 137.94 & 1.64 \\
\hline 23.10 & 0.11 & 75.27 & 0.81 & 138.22 & 0.57 \\
\hline 23.48 & 0.34 & 75.82 & 1.66 & 138.60 & 0.54 \\
\hline 24.04 & 0.01 & 76.37 & 0.31 & 139.44 & 0.28 \\
\hline 24.60 & 0.38 & 76.77 & 0.14 & 139.72 & 0.14 \\
\hline 25.00 & 0.14 & 80.92 & 0.41 & 140.10 & 0.14 \\
\hline 25.54 & 7.33 & 81.22 & 1.37 & 140.94 & 0.38 \\
\hline 26.10 & 5.88 & 81.90 & 9.96 & 141.22 & 0.54 \\
\hline 26.50 & 8.84 & 82.42 & 1.84 & 141.60 & 0.90 \\
\hline 27.04 & 3.11 & 82.72 & 1.13 & 142.44 & 0.11 \\
\hline 30.91 & 1.69 & 83.92 & 0.22 & 142.72 & 0.47 \\
\hline 32.41 & 3.28 & 84.22 & 0.40 & 143.10 & 0.27 \\
\hline 37.68 & 1.00 & 84.90 & 0.09 & 143.94 & 0.85 \\
\hline 38.12 & 1.58 & 85.42 & 0.34 & 144.22 & 0.52 \\
\hline 38.60 & 0.13 & 89.27 & 3.91 & 147.98 & 0.05 \\
\hline 39.14 & 5.26 & 89.92 & 1.27 & 149.30 & 0.00 \\
\hline 39.62 & 7.33 & 90.12 & 1.57 & 152.68 & 0.01 \\
\hline 40.10 & 3.58 & 90.77 & 0.27 & 156.86 & 0.00 \\
\hline 40.76 & 0.75 & 91.42 & 0.54 & 184.67 & 0.00 \\
\hline 41.12 & 18.29 & 91.62 & 0.63 & 186.17 & 0.05 \\
\hline 41.52 & 4.35 & 92.27 & 0.06 & 187.67 & 0.01 \\
\hline 43.56 & 1.85 & 92.92 & 0.07 & 194.35 & 0.11 \\
\hline 44.02 & 12.44 & 93.12 & 0.28 & 194.92 & 0.16 \\
\hline 44.72 & 12.92 & 93.77 & 0.49 & 195.42 & 0.00 \\
\hline 45.10 & 10.87 & 94.45 & 0.69 & 195.85 & 0.00 \\
\hline 45.56 & 9.59 & 94.62 & 0.63 & 196.92 & 0.06 \\
\hline 49.50 & 4.21 & 95.27 & 1.48 & 197.35 & 0.00 \\
\hline 50.02 & 4.90 & 95.95 & 0.15 & 197.92 & 0.01 \\
\hline 50.78 & 4.81 & 96.12 & 0.27 & 198.42 & 0.00 \\
\hline 51.00 & 1.39 & 96.77 & 0.07 & 198.85 & 0.00 \\
\hline 51.62 & 3.06 & 100.26 & 0.54 & 199.42 & 0.00 \\
\hline 52.28 & 0.47 & 100.92 & 0.21 & 200.35 & 0.00 \\
\hline 52.78 & 2.97 & 101.47 & 0.32 & 200.92 & 0.04 \\
\hline
\end{tabular}

The first minor occurrence of ice-rafted debris at Hole 701B was at about $8.78 \mathrm{Ma}$ (200.92 mbsf), which is the oldest available sample in this hole (Fig. 3). Little ice-rafted debris was identified in any of the other examined samples up to $8.5 \mathrm{Ma}$. The first minor influx of more than a few grains of ice-rafted debris at Site 699 occurred at about $8.87 \mathrm{Ma}$. There is a hiatus at both sites in the upper Miocene (Site 699, 6.0-10.0 Ma; Site 701, 6.35-7.60
$\mathrm{Ma}$ ), which may be age equivalent with a major erosional phase on the Maurice Ewing Bank from about 7.2 to $6.3 \mathrm{Ma}$ due to a more vigorous Antarctic Circumpolar Current (ACC) (Ciesielski et al., 1982). Bornhold (1983) dated an interval of high ice-rafted debris accumulation rates at Deep Sea Drilling Project (DSDP) Site 513 (Fig. 1) as between 6.7 and $6.5 \mathrm{Ma}$, but this interval is missing at Sites 699 and 701 . 
Table 3. Amount of coarse-grained ice-rafted debris, Hole 704A.

\begin{tabular}{|c|c|c|c|}
\hline $\begin{array}{l}\text { Sample depth } \\
\text { (mbsf) }\end{array}$ & $\begin{array}{l}\text { Coarse-grained } \\
\text { ice-rafted debris } \\
(\mathrm{mg} / \mathrm{g})\end{array}$ & $\begin{array}{l}\text { Sample depth } \\
\text { (mbsf) }\end{array}$ & $\begin{array}{l}\text { Coars-grained } \\
\text { ice-rafted debris } \\
(\mathrm{mg} / \mathrm{g})\end{array}$ \\
\hline 0.72 & 0.61 & 118.42 & 0.00 \\
\hline 2.22 & 0.00 & 119.92 & 0.50 \\
\hline 3.72 & 1.31 & 120.82 & 0.00 \\
\hline 5.22 & 0.00 & 121.92 & 0.61 \\
\hline 6.72 & 0.00 & 123.42 & 0.40 \\
\hline 7.86 & 0.00 & 124.92 & 0.00 \\
\hline 9.36 & 0.00 & 126.42 & 0.86 \\
\hline 10.86 & 0.10 & 127.92 & 0.00 \\
\hline 12.36 & 0.00 & 129.42 & 1.28 \\
\hline 13.86 & 0.11 & 131.42 & 0.17 \\
\hline 15.36 & 0.86 & 132.92 & 0.39 \\
\hline 16.86 & 0.11 & 134.42 & 0.00 \\
\hline 17.68 & 0.00 & 135.92 & 0.00 \\
\hline 19.18 & 0.00 & 137.42 & 0.00 \\
\hline 20.68 & 0.98 & 138.92 & 0.00 \\
\hline 22.18 & 1.68 & 139.82 & 0.00 \\
\hline 23.68 & 0.36 & 140.92 & 0.00 \\
\hline 25.18 & 1.19 & 142.42 & 0.00 \\
\hline 29.90 & 0.35 & 143.92 & 0.56 \\
\hline 34.10 & 0.00 & 145.42 & 0.00 \\
\hline 39.01 & 0.24 & 146.92 & 0.00 \\
\hline 42.01 & 0.00 & 148.42 & 0.00 \\
\hline 43.51 & 0.10 & 150.42 & 2.39 \\
\hline 45.90 & 0.00 & 151.92 & 0.00 \\
\hline 47.40 & 3.09 & 153.42 & 0.29 \\
\hline 48.90 & 0.00 & 154.92 & 0.10 \\
\hline 50.40 & 0.09 & 156.42 & 0.00 \\
\hline 51.90 & 0.00 & 157.92 & 0.00 \\
\hline 53.40 & 0.00 & 159.92 & 0.00 \\
\hline 54.50 & 0.00 & 161.42 & 0.22 \\
\hline 56.92 & 0.00 & 162.92 & 0.74 \\
\hline 58.40 & 0.00 & 164.42 & 0.46 \\
\hline 59.93 & 0.17 & 165.92 & 0.00 \\
\hline 61.42 & 0.00 & 167.42 & 3.90 \\
\hline 62.95 & 0.00 & 169.42 & 0.50 \\
\hline 64.91 & 0.00 & 170.92 & 0.00 \\
\hline 66.41 & 0.00 & 172.42 & 0.00 \\
\hline 67.91 & 0.28 & 173.92 & 0.70 \\
\hline 69.41 & 0.00 & 175.42 & 0.06 \\
\hline 70.91 & 0.06 & 176.92 & 0.67 \\
\hline 72.41 & 0.50 & 179.12 & 0.00 \\
\hline 74.41 & 0.00 & 186.62 & 0.00 \\
\hline 75.91 & 0.00 & 182.12 & 0.00 \\
\hline 77.41 & 0.00 & 183.62 & 0.00 \\
\hline 78.91 & 0.00 & 185.12 & 1.11 \\
\hline 80.41 & 0.87 & 186.62 & 0.00 \\
\hline 81.91 & 0.14 & 187.80 & 0.00 \\
\hline 82.81 & 0.09 & 189.30 & 0.00 \\
\hline 83.90 & 0.05 & 190.80 & 0.00 \\
\hline 85.40 & 0.34 & 192.30 & 0.00 \\
\hline 86.90 & 0.60 & 197.61 & 0.00 \\
\hline 88.40 & 0.23 & 199.11 & 0.08 \\
\hline 89.90 & 0.00 & 200.61 & 0.00 \\
\hline 91.40 & 0.11 & 202.11 & 0.01 \\
\hline 92.90 & 0.00 & 203.61 & 0.01 \\
\hline 93.42 & 0.42 & 208.00 & 0.36 \\
\hline 94.92 & 0.00 & 209.50 & 0.09 \\
\hline 96.42 & 0.00 & 211.00 & 0.38 \\
\hline 97.92 & 0.36 & 212.50 & 0.00 \\
\hline 99.35 & 0.21 & 214.00 & 0.01 \\
\hline 100.92 & 0.00 & 217.50 & 0.02 \\
\hline 101.82 & 3.24 & 219.00 & 0.00 \\
\hline 102.92 & 0.00 & 220.50 & 0.00 \\
\hline 104.42 & 0.00 & 222.00 & 0.00 \\
\hline 105.92 & 0.90 & 223.50 & 0.00 \\
\hline 107.42 & 0.52 & 226.72 & 0.00 \\
\hline 108.92 & 0.00 & 228.22 & 0.01 \\
\hline 110.42 & 0.00 & 229.72 & 0.00 \\
\hline 112.42 & 0.73 & 231.22 & 0.00 \\
\hline 113.92 & 0.37 & 232.72 & 0.00 \\
\hline 115.42 & 0.39 & 234.22 & 0.00 \\
\hline 116.92 & 0.36 & & \\
\hline
\end{tabular}

Table 4. Hole 699A age model.

\begin{tabular}{|c|c|c|}
\hline $\begin{array}{l}\text { Depth }^{\mathrm{a}} \\
\text { (mbsf) }\end{array}$ & $\begin{array}{l}\text { Age } \\
\text { (Ma) }\end{array}$ & Event \\
\hline 0.21 & 0.195 & LAAD Hemidiscus karstenii \\
\hline 4.90 & 0.420 & FAAD Hemidiscus karstenii \\
\hline 8.16 & 0.620 & LAD Actinocyclus ingens \\
\hline 10.59 & 0.730 & Brunhes/Matuyama \\
\hline 11.17 & 0.770 & Estimate based on sedimentation rate above hiatus \\
\hline 11.17 & 1.140 & ${ }^{b}$ Estimate based on sedimentation rate below hiatus \\
\hline 11.75 & 1.200 & Top Actinocyclus ingens acme $1(>10 \%)$ \\
\hline 19.59 & 1.660 & Top Olduvai \\
\hline 21.19 & 1.880 & Base Olduvai \\
\hline 29.45 & 2.470 & Matuyama/Gauss \\
\hline 31.74 & 2.630 & Top Nitzschia weaveri acme $2(>10 \%)$ \\
\hline 36.50 & 2.990 & Base Cosmiodiscus insignis acme $(>10 \%)$ \\
\hline 39.03 & 3.180 & First consistent Coscinodiscus vulnificus \\
\hline 40.69 & 3.400 & Gauss/Gilbert \\
\hline 42.82 & 3.830 & First consistent Nitzschia interfrigidaria \\
\hline 44.00 & 3.880 & Top Cochiti \\
\hline 45.27 & 4.190 & FAD Nitzschia angulata \\
\hline 45.27 & 4.690 & LAD Dictyocha pygmaea \\
\hline 53.61 & 5.350 & Gilbert/C3AN \\
\hline 58.00 & 5.530 & C 3 AN. 33 \\
\hline 61.00 & 5.680 & C3AN.61 \\
\hline 62.40 & 5.890 & C3AN/C3AR \\
\hline 64.64 & 6.000 & ${ }^{c}$ Estimate \\
\hline 64.64 & 10.000 & Estimate, within Chron $\mathrm{C} 5 \mathrm{~N}$, lower to middle part \\
\hline 66.59 & 10.420 & based on abundant Denticulopsis dimorpha \\
\hline 66.59 & 14.080 & ${ }^{d}$ Very crude; only one sample in this interval \\
\hline 68.36 & 14.300 & \\
\hline 68.36 & 23.500 & Estimate, only slightly younger than $23.55 \mathrm{Ma}$ \\
\hline 70.29 & 23.550 & C6CN.3? \\
\hline 94.74 & 24.210 & $\mathrm{C} 6 \mathrm{CN} / \mathrm{C} 6 \mathrm{CR}$ \\
\hline
\end{tabular}

Note: $\mathrm{LAAD}=$ last abundant appearance datum; FAAD = first abundant appearance datum; $\mathrm{LAD}=$ last-appearance datum; $\mathrm{FAD}=$ firstappearance datum.

${ }^{\text {a }}$ Repeated entries signify hiatuses.

b Between 1.20 and $1.66 \mathrm{Ma}$.

In C3AR, but younger than $6.00 \mathrm{Ma}$ based on correlation to Hole 704B.

d Sample is middle Miocene, probably lower Nitzschia denticuloides Zone.

There was a major influx of ice-rafted debris at Site 699 in latest Miocene to early Gilbert Chron time (Fig. 2), corresponding to a period of general climatic cooling and glacial expansion from 6.0 to 5.2 Ma (Loutit and Kennett, 1979) and perhaps correlated with the first appearance of ice-rafted debris at DSDP Site 344 off Spitsbergen (Warnke, 1982). This interval is also characterized by minor influxes of ice-rafted debris at Site 701 at 5.88 and $5.59 \mathrm{Ma}$ (Fig. 3). These peaks may be comparable with Bornhold's (1983) interval B (5.5 to $5.0 \mathrm{Ma})$ of very low icerafted debris accumulation rates (Fig. 5).

\section{Pliocene}

Another significant ice-rafted debris peak at Site 701 occurred at $4.85 \mathrm{Ma}$ as ice rafting continued, but rates of accumulation remained at low levels until about $4.4 \mathrm{Ma}$ within a hiatus identified at Site 699 . These low levels may be due to the deterioration of the West Antarctic Ice Sheet (Bornhold, 1983 ) in response to warmer climatic conditions during the early Pliocene from 4.8 to $4.3 \mathrm{Ma}$ (Ciesielski and Weaver, 1974), when sea-surface temperatures were $5^{\circ}-10^{\circ} \mathrm{C}$ warmer than today in the Southern Ocean (Ciesielski et al., 1982). This peak and the one described in the following may well correspond to the peak identified by Anderson (1985) for this time interval.

Very little or no ice-rafted debris was deposited in the lower part of the sedimentary column of Site 704 until the middle Pliocene (4.19 Ma). Site 699 AMARs are variable from 3.86 to $3.32 \mathrm{Ma}$, with the first significant influx of ice-rafted debris at $3.86 \mathrm{Ma}$, which correlates very well with the cluster of data points and minor $\left(<10 \mathrm{mg} / \mathrm{cm}^{2} / 10^{3} \mathrm{yr}\right)$ influx of 
Table 5. Site 701 age model.

\begin{tabular}{|c|c|c|}
\hline $\begin{array}{c}\text { Depth }^{\mathrm{a}} \\
\text { (mbsf) }\end{array}$ & $\begin{array}{l}\text { Age } \\
\text { (Ma) }\end{array}$ & Event \\
\hline \multicolumn{3}{|l|}{ Hole 701A } \\
\hline 17.02 & 0.73 & Brunhes/Matuyama \\
\hline 31.50 & 0.91 & Top Jaramillo (as recognized in Hole 701C) \\
\hline 32.50 & 1.20 & Top Actinocyclus ingens acme (as recognized in Hole 701C) \\
\hline 37.00 & 1.45 & Base Actinocyclus ingens acme (as recognized in Hole 701C) \\
\hline 44.93 & 1.66 & Top Olduvai \\
\hline 50.95 & 1.88 & Base Olduvai \\
\hline 68.73 & 2.47 & Matuyama/Gauss \\
\hline 71.40 & 2.63 & LAAD (>10\%) Nitzschia weaveri (top acme 2) \\
\hline 71.40 & 3.11 & Last consistent Nitzschia interfrigidaria (s. str.) \\
\hline \multicolumn{3}{|l|}{ Hole 701C } \\
\hline 74.48 & 3.40 & Gauss/Gilbert \\
\hline 99.05 & 4.10 & Top Nunivak \\
\hline 102.97 & 4.24 & Base Nunivak (based on Hole 701B) \\
\hline 106.83 & 4.40 & Top Sidufjall (based on Hole 701B) \\
\hline 110.59 & 4.47 & Base Sidufjall (based on Hole 701B) \\
\hline 113.99 & 4.57 & Top Thvera (based on Hole 701B) \\
\hline 116.10 & 4.77 & Base Thvera \\
\hline 140.27 & 5.68 & C3AN.61 \\
\hline 144.20 & 5.89 & C3AN/C3AR \\
\hline 165.54 & 6.36 & FAD Cosmiodiscus insignis $\mathrm{v}$, triangulata \\
\hline 174.27 & 6.55 & $\begin{array}{l}\text { Age above hiatus based on sedimentation between } 114.20 \\
\text { and } 165.54 \text { mbsf extrapolated to hiatus depth. }\end{array}$ \\
\hline 174.27 & 7.60 & $\begin{array}{l}\text { Age of hiatus based on sedimentation rate between } 7.90 \text { and } \\
8.21 \text { Ma extrapolated to hiatus depth }\end{array}$ \\
\hline 179.07 & 7.90 & $\mathrm{C} 4 \mathrm{R} / \mathrm{C} 4 \mathrm{AN}$ \\
\hline 183.98 & 8.21 & C4AN.52 \\
\hline 188.24 & 8.41 & C4AN.50 \\
\hline 194.76 & 8.50 & C4AN/C4AR \\
\hline 204.02 & 8.92 & C4AR/C5N \\
\hline 228.40 & 10.06 & C5N/C5R \\
\hline 234.18 & 11.36 & LAD Cyrtocapsella tetrapera \\
\hline
\end{tabular}

Note: Values given from Holes $701 \mathrm{~A}$ and $701 \mathrm{C}$, except as noted. Hole 701A has not been studied in sufficient detail to determine depths of unconformities, which are therefore inferred using data from Hole 701C. There may be some difference, as the Brunhes/Matuyama boundary is shallower in Hole $701 \mathrm{~A}(17.02 \mathrm{vs} .23 .00 \mathrm{mbsf})$ and the Matuyama/Gauss boundary is deeper ( 68.73 vs. $66.70 \mathrm{mbsf}$ ), which may alter the ice-rafted debris data near hiatuses. The hiatus at $71.40 \mathrm{mbsf}$ is inferred as the midpoint between bracketing datums 71.63 and $71.17 \mathrm{mbsf}$ in Hole 701C. The hiatus at $174.27 \mathrm{mbsf}$ is inferred as the midpoint between bracketing samples from 178.30 and 170.23 mbsf (P. F. Ciesielski, pers. comm., 1990). LAAD = last abundant appearance datum; $\mathrm{LAD}=$ last-appearance datum; $\mathrm{FAD}=$ first-appearance datum

${ }^{\text {a }}$ Repeated entries signify hiatuses.

ice-rafted debris at $3.82 \mathrm{Ma}$. This occurred during a major cooling trend from 4.2 to 3.0 Ma (Ciesielski and Weaver, 1974) when the West Antarctic Ice Sheet may have been reestablished (Bornhold, 1983) and formation of the Northern Hemisphere ice sheets began (Kennett, 1977; Shackleton et al., 1984). It may correspond to Bornhold's (1983) interval C of increased ice-rafted debris accumulation rates from 4.1 to 3.86 Ma with a peak at $4.01 \mathrm{Ma}$. The peak of ice-rafted debris at Site 701 at $3.61 \mathrm{Ma}$ corresponds to a peak at Site 699 at precisely the same time (Fig. 5).

Between 3.23 and $2.58 \mathrm{Ma}$ is another period of variable ice-rafted debris AMARs at Site 699 with a minor influx of ice-rafted debris at $3.14 \mathrm{Ma}$, which is perhaps equivalent to a cluster of data points at Site 701 (centered around 3.3 Ma). The next minor peak at Site 704 occurs at $2.95 \mathrm{Ma}$ coincident with the initiation of Northern Hemisphere ice rafting (Janssen et al., 1988). This occurred during a period of generally warm conditions (Ciesielski et al., 1982). Bornhold (1983) found relatively low concentrations of ice-rafted debris for this time interval at DSDP Leg 71 sites. This pattern somewhat follows the model in which a sustained glacial interval would be bracketed at intermediate latitudes by ice-rafted debris peaks at the beginning and end of a cool period (Keany et al., 1976).

Between 2.63 and $2.47 \mathrm{Ma}$ a period of low sedimentaccumulation rates occurred at Sites 699 and 701 ; this same time period contains glacial interval D of Bornhold (1983) with a maximum at $2.61 \mathrm{Ma}$. The cool conditions beginning at this time continued until about 1.0 Ma (Keany and Kennett, 1972). The initiation of these cool conditions is marked by a dramatic increase in ice-rafted debris AMARs at all three sites. The highest AMAR for Site 699 occurs at $2.38 \mathrm{Ma}$ compared with $2.46 \mathrm{Ma}$ at Site 704 and $2.24 \mathrm{Ma}$ at Site 701. Resolution of these differences, however, is not possible at present because of the sample spacing. We consider these maxima to be time equivalent. The high rates indicated for these sites are attributed to large-scale "wetbased" glaciations on Antarctica. It has been suggested that these AMAR peaks are linked to the glaciations that produced the Sirius tills and are tentatively named "Sirius till equivalent, marine" (STEM) (Warnke and Allen, this volume). These correlations are tentative, however, and await definite age determinations of these tills.

Ice-rafted debris accumulation rates at Site 704 varied greatly over the next $0.2 \mathrm{~m}$.y. with a major flux at $2.29 \mathrm{Ma}$ while cool conditions continued. Fluctuating, but significant, influxes of ice-rafted debris occurred between 2.11 and 1.79 $\mathrm{Ma}$. Another grouping of data points at Site 701 occurred between 2.07 and $1.83 \mathrm{Ma}$, which corresponds fairly well with the interval of variable but increased ice-rafted debris concentrations from 2.02 to $1.86 \mathrm{Ma}$ at Site 699, Bornhold's (1983) 
Table 6. Hole 704A age model.

\begin{tabular}{rrl}
\hline $\begin{array}{r}\text { Depth } \\
\text { (mbsf) }\end{array}$ & $\begin{array}{c}\text { Age } \\
\text { (Ma) }\end{array}$ & Event \\
\hline 1.14 & 0.195 & LAAD Hemidiscus karstenii \\
3.52 & 0.258 & FAD Emiliana huxleyi, mid-stage 8 \\
7.64 & 0.423 & FAAD Hemidiscus karstenii \\
8.65 & 0.460 & Last consistent Pseudoemiliana lacunosa \\
13.25 & 0.620 & LAD Actinocyclus ingens \\
34.51 & 0.730 & Brunhes/Matuyama \\
38.40 & 0.910 & Top Jaramillo \\
44.27 & 0.980 & Base Jaramillo \\
89.09 & 1.660 & Top Olduvai \\
168.45 & 2.470 & Matuyama/Gauss \\
176.10 & 2.920 & Top Kaena \\
177.80 & 2.990 & Base Kaena \\
179.10 & 3.080 & Top Mammoth \\
181.50 & 3.180 & Base Mammoth \\
186.65 & 3.400 & Gauss/Gilbert \\
198.55 & 3.880 & Top Cochiti \\
201.95 & 3.970 & Base Cochiti \\
204.20 & 4.100 & Top Nunivak \\
210.35 & 4.240 & Base Nunivak \\
212.00 & 4.400 & Top Sidufjall \\
213.25 & 4.470 & Base Sidufjall \\
215.40 & 4.570 & Top Thvera \\
219.45 & 4.770 & Base Thvera \\
224.76 & 5.350 & Gilbert/C3AN \\
\hline
\end{tabular}

Note: $\mathrm{LAAD}=$ last abundant appearance datum; FAAD $=$ first abundant appearance datum; $L A D=$ last-appearance datum; FAD = first-appearance datum.

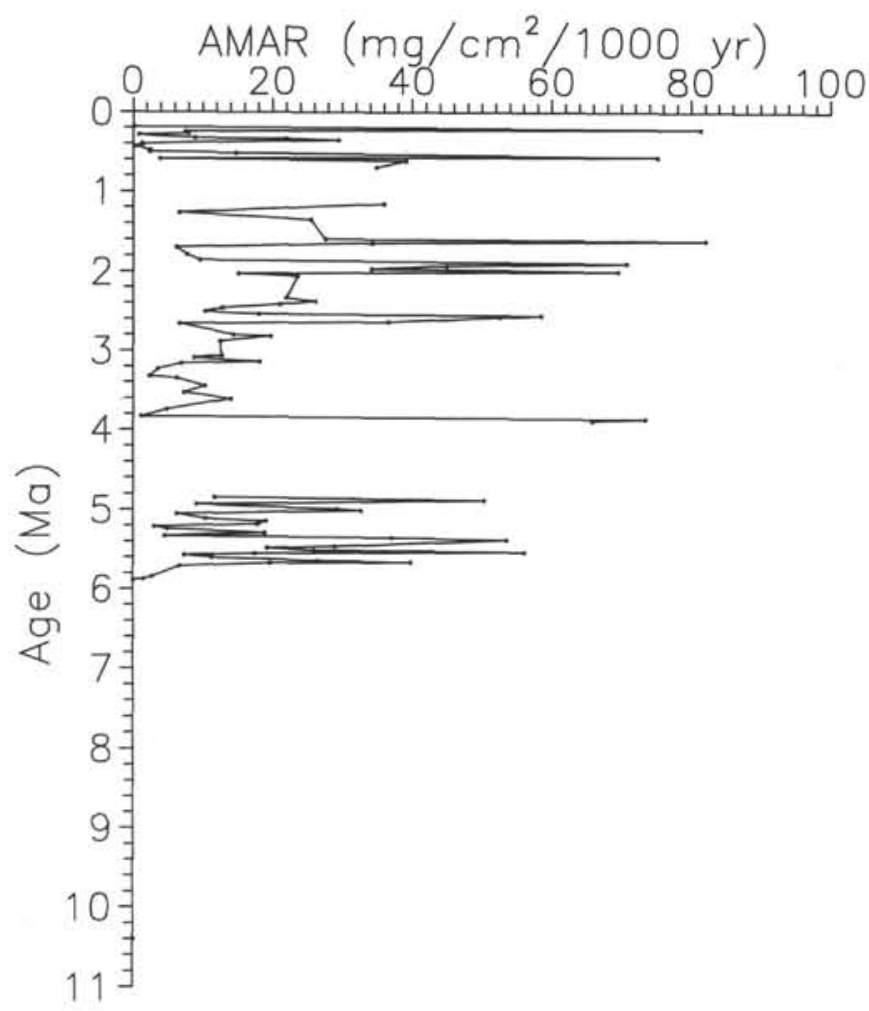

Figure 2. Variations in ice-rafted debris apparent mass accumulation rates during the past $11.0 \mathrm{Ma}$ at Hole $699 \mathrm{~A}$.

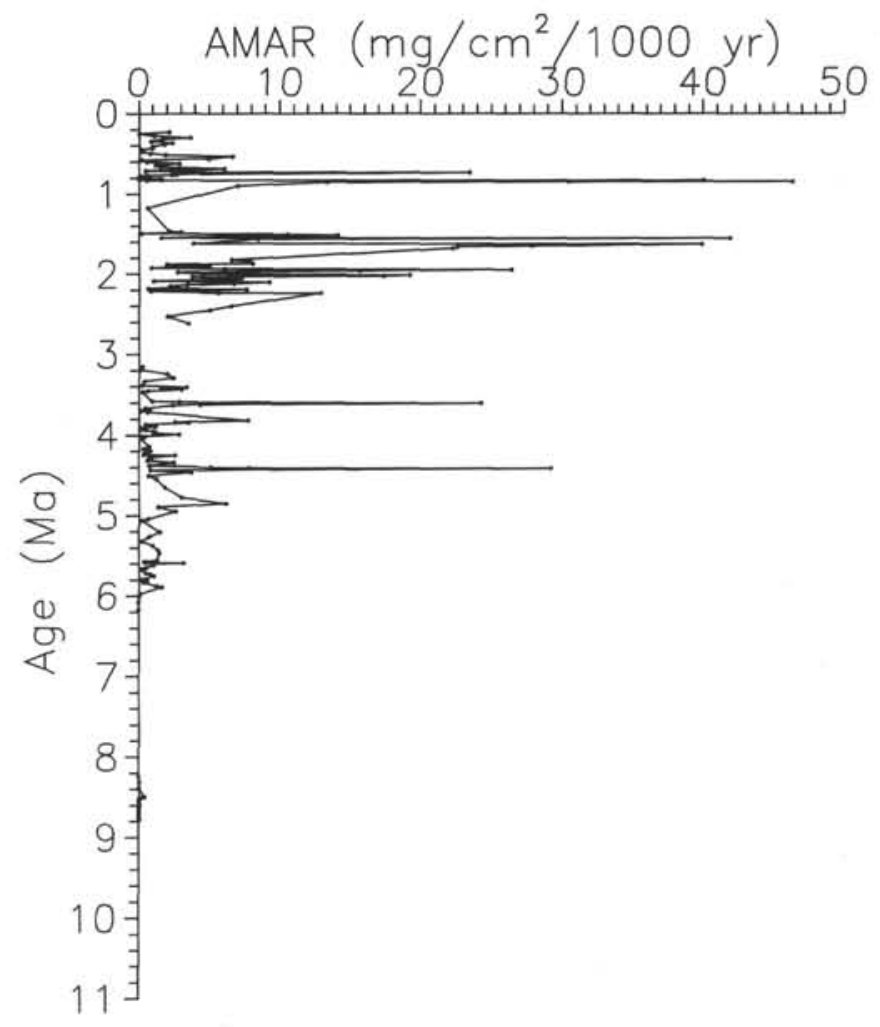

Figure 3. Variations in ice-rafted debris apparent mass accumulation rates during the past $11.0 \mathrm{Ma}$ at Site 701 .

interval E (2.1 to $1.8 \mathrm{Ma}$ ), the age of the thickest glacial till in Patagonia dated between 2.10 to $1.91 \mathrm{Ma}$ (Fig. 5; Fleck et al., 1972), and the suggested "end of STEM" at about $1.77 \mathrm{Ma}$ (Warnke and Allen, this volume).

\section{Pleistocene}

The next grouping of AMAR peaks at Site 701, from 1.64 to $1.52 \mathrm{Ma}$, is perhaps equivalent to the peak at $1.62 \mathrm{Ma}$ at Site 699 and a corresponding significant peak at Site 704 (1.53 Ma). As at Site 699 at $1.36 \mathrm{Ma}$, there is only a minor influx of ice-rafted debris at Site 701 indicated at 1.47 and $1.48 \mathrm{Ma}$. These influxes coincide with Bornhold's (1983) interval F of elevated ice-rafted debris accumulation rates, occurring from 1.5 to $0.97 \mathrm{Ma}$, and perhaps the most severe glacial phase in Patagonia between 1.3 and 1.0 Ma (Fig. 5; Mercer, 1976).

Another major peak in the ice-rafted debris AMAR of Site 704 at $1.03 \mathrm{Ma}$ was not recorded at Site 701 because of a possible hiatus at about 32 mbsf (not shown on Fig. 3). The major influxes of ice-rafted debris at Site 701 from 0.85 to 0.84 Ma coincide again with the end of a period of cool conditions (Keany and Kennett, 1972). Moderate ice-rafted debris AMARs at Site 699 characterize the intervals bracketing the hiatus, with a significant peak above the hiatus at $0.57 \mathrm{Ma}$ (Fig. 2). The next grouping of data points at Site 704 occurred between 600,000 and 800,000 yr B.P. and corresponds to significant groups of peaks at Sites 699 and 701 . These groups compare with interval G from 0.90 to $0.65 \mathrm{Ma}$ (Fig. 5), which is characterized by very high rates of ice-rafted debris accumulation at DSDP Site 514 (Bornhold, 1983), as well as on the Maurice Ewing Bank (Ciesielski et al., 1982) and at other study areas in the Southern Ocean (Ledbetter and Watkins, 1978). This interval of greater ice-rafted debris influx is, according to Bornhold (1983), more likely due to changing 


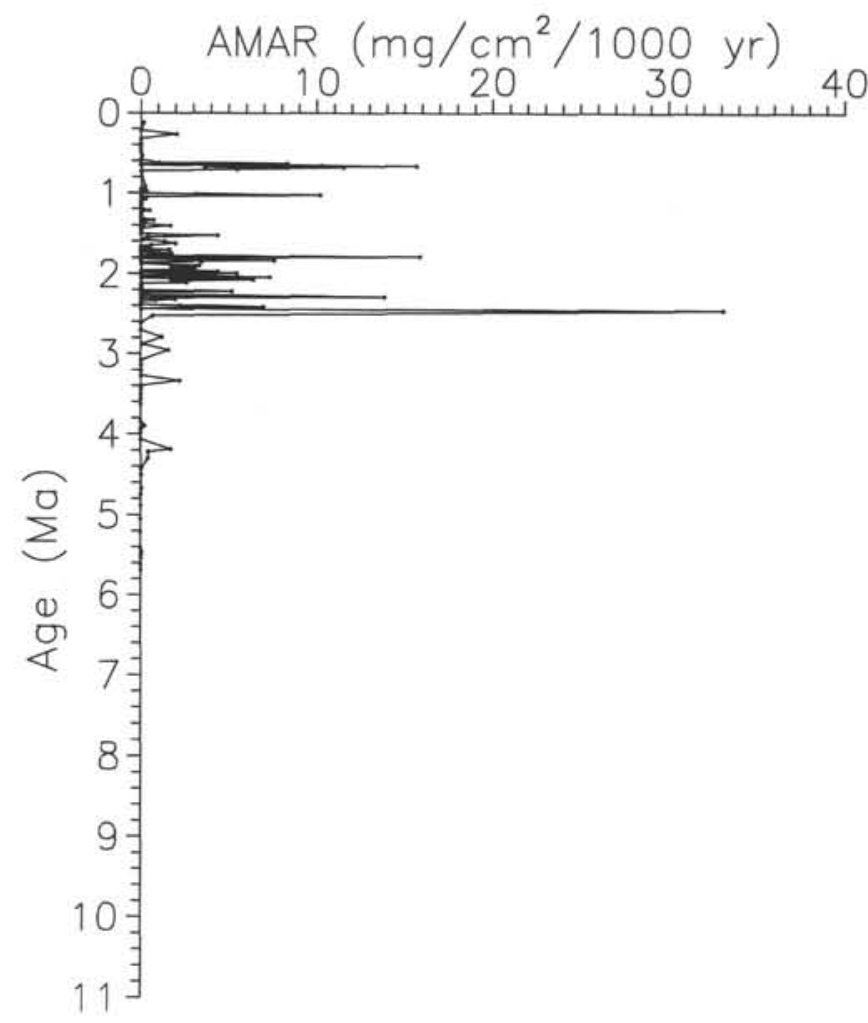

Figure 4. Variations in ice-rafted debris apparent mass accumulation rates during the past $11.0 \mathrm{Ma}$ at Hole $704 \mathrm{~A}$.

circulation patterns and increased rates of iceberg melting than to increased Antarctic glaciation.

Above the $600,000 \mathrm{Ma}$ level, ice-rafted debris AMARs at Site 701 were highly variable with a few minor peaks, but followed a generally decreasing trend. Accumulation rates at Site 704 dropped off drastically until the last minor peak during isotopic stage 7. At Site 699 the last significant peak of ice-rafted debris occurred at $0.22 \mathrm{Ma}$. These general trends for Sites 701 and 704 do not correspond well with Bornhold's (1983) interval H (0.40 Ma to the Holocene, Fig. 5), described as having a sawtooth pattern with a general increase in rates of ice-rafted debris accumulation, or with moderated climatic conditions leading to a less vigorous ACC and renewed deposition on Maurice Ewing Bank (Ciesielski et al., 1982), although such a trend may be discernible at Site 699 (noting, however, the sample spacing). These peaks are at least in part equivalents of the large, late Pleistocene Northern Hemisphere ice sheets.

\section{CONCLUSIONS}

Ice-rafted debris first arrived at about 23.5 Ma (only identified at Site 699); however, significant amounts did not reach the northeast slope of the Northeast Georgia Rise until the late Miocene. At Site 701 , as previously reported by Bornhold (1983) for sites south of the Antarctic Convergence, ice-rafted debris accumulation rates remained low until the early Pliocene ( $\sim .5 \mathrm{Ma})$. In contrast, Site 699 showed strong pulses of ice rafting. Accumulation rates at Site 704 north of the Polar Front remained low until about $2.46 \mathrm{Ma}$, when the greatest influx of ice-rafted debris occurred. The periods of increased ice-rafted debris AMARs at the Leg 114 sites correlate reasonably well with five of the eight ice-rafting intervals identified by Bornhold (1983) for Leg 71 sites, although the biostratigraphy of these sites must be reevaluated in light of new age models.

The high AMARs commencing at about $2.5 \mathrm{Ma}$ at Sites 699,701 , and 704 at tentatively attributed to large-scale wet-based glaciations, which may have produced the Sirius tills on Antarctica. This suggestion must await confirmation of the age of this group of tills on Antarctica. In any event, these wet-based conditions continued until about $1.77 \mathrm{Ma}$, when ice rafting significantly decreased, at least as defined at Site 704. While greater influxes of ice-rafted debris into the study area occurred during periods of colder conditions, there is some evidence lending support to the proposed model of elevated ice-rafted debris accumulation rates marking the beginning and end of a cool period (diachronous deposition) (Keany et al., 1976) and of decreasing ice-rafted debris flux as boundary conditions on Antarctica changed (Warnke, 1970).

Although great progress has been made, better stratigraphic control leading to more accurate age determinations and sedimentation rates would improve the results obtained. Variations in ocean circulation patterns and the paths followed by icebergs between glacial phases must also affect the influx of ice-rafted debris at various locations in the Southern Ocean. Perhaps sites drilled along these iceberg tracks or at least along a north-south track would also lead to better understanding of climatic variations as distance from the continent increases.

\section{ACKNOWLEDGMENTS}

We express our appreciation to Paul F. Ciesielski, Brian D. Bornhold, and Michael T. Ledbetter for critical review of the manuscript and the many valuable suggestions for its improvement. A post-cruise grant from USSAC is gratefully acknowledged.

\section{REFERENCES}

Allen, C. P., 1989. History of ice-rafting at ODP Leg 114 sites, Subantarctic/South Atlantic [M.S. thesis]. Calif. State Univ., Hayward.

Anderson, D. M., 1985. Pliocene paleoceanography of the Southern Ocean and the development of the West Antarctic Ice Sheet [M.S. thesis]. San Jose State Univ., San Jose, CA.

Anderson, J. B., Brake, C., Domack, E., Myers, N., and Wright, R., 1983. Development of a polar glacial-marine sedimentation model from Antarctic Quaternary deposits and glaciological information. In Molnia, B. F. (Ed.), Glacial-Marine Sedimentation: New York (Plenum Press), 233-264.

Barker, P. F., and Burrell, J., 1982. The influence upon Southern Ocean circulation, sedimentation, and climate of the opening of the Drake Passage. In Craddock, C. (Ed.), Antarctic Geoscience: Madison (Univ. of Wisconsin), 377-385.

Bornhold, B. D., 1983. Ice-rafted debris in sediments from Leg 71, southwest Atlantic Ocean. In Ludwig, W. J., Krasheninnikov, V. A., et al., Init. Repts. DSDP, 71: Washington (U.S. Govt. Printing Office), 307-316.

Ciesielski, P. F., Ledbetter, M. T., and Ellwood, B. B., 1982. The development of Antarctic glaciation and the Neogene paleoenvironment of the Maurice Ewing Bank. Mar. Geol., 46:1-51.

Ciesielski, P. F., and Weaver, F. M., 1974. Early Pliocene temperature changes in the Antarctic Seas. Geology, 12:511-515.

Fleck, R. J., Mercer, J. H., Nairn, A.E.M., and Peterson, D. N., 1972. Chronology of late Pliocene and early Pleistocene glacial and magnetic events in southern Argentina. Earth Planet. Sci. Lett., $16: 15-22$.

Janssen, E., Bleil, U., Henrich, R., Kringstad, L., and Slettemark, B., 1988. Paleoenvironmental changes in the Norwegian Sea and the northeast Atlantic during the last 2.8 m.y.: Deep Sea Drilling Project/Ocean Drilling Program Sites 610, 642, 643, and 644. Paleoceanography, 3:563-581. 
Keany, J., and Kennett, J. P., 1972. Pliocene-early Pleistocene palaeoclimatic history recorded in Antarctic-Subantarctic deepsea cores. Deep-Sea Res., Part A, 19:529-548.

Keany, J., Ledbetter, M. T., Watkins, N. D., and Huang, T. C., 1976. Diachronous deposition of ice-rafted debris in sub-Antarctic deepsea sediments. Geol. Soc. Am. Bull., 87:873-882.

Kennett, J. P., 1977. Cenozoic evolution of Antarctic glaciation, the circum-Antarctic Ocean, and their impact on global paleoceanography. J. Geophys. Res., 82:3843-3860.

Ledbetter, M. T., and Watkins, N. D., 1978. Separation of primary ice-rafted debris from lag deposits, utilizing manganese micronodule accumulation rates in abyssal sediments of the Southern Ocean. Geol. Soc. Am. Bull., 89:1619-1629.

Loutit, T. S., and Kennett, J. P., 1979. Application of carbon isotope stratigraphy to late Miocene shallow marine sediments, New Zealand. Science, 204:1196-1199.

Mercer, J. H., 1976. Glacial history of southernmost South America. Quat. Res., 6:125-166.

Molnia, B. F., 1983. Distal glacial-marine sedimentation: abundance, composition, and distribution of North Atlantic Ocean Pleistocene ice-rafted sediment. In Molnia, B. F. (Ed.), Glacial-Marine Sedimentation: New York (Plenum Press), 593-625.
Plafker, G., Bartsch-Winkler, S., and Ovenshine, A. T., 1977. Paleoglacial implications of coarse detritus in DSDP Leg 36 cores. In Barker, P. F., Dalziel, I.W.D., et al., Init. Repts. DSDP, 36: Washington (U.S. Govt. Printing Office), 857-864.

Shackleton, N. J., Backman, J., Zimmerman, H., Kent, D. V., Hall, M. A., Roberts, D. G., Schnitker, D., Baldauf, J., Desprairies, A., Homrighausen, R., Huddlestun, P., Keene, J. B., Kaltenback, A. J., Krumsiek, K.A.O., Morton, A. C., Murray, J. W., and Westberg-Smith, J., 1984. Oxygen isotope calibration of the onset of ice-rafting in DSDP Site 552A: history of glaciation in the North Atlantic region. Nature, 307:620-623.

Warnke, D. A., 1970. Glacial erosion, ice rafting, and glacial-marine sediments: Antarctica and the Southern Ocean. Am. J. Sci., 269:276-294.

1982. Pre-middle Pliocene sediments of glacial and periglacial origin in the Norwegian-Greenland Seas: results of D.S.D.P. Leg 38. Earth Evol. Sci., 2:69-78.

Date of initial receipt: 17 April 1989

Date of acceptance: 25 January 1990

Ms 114B-118
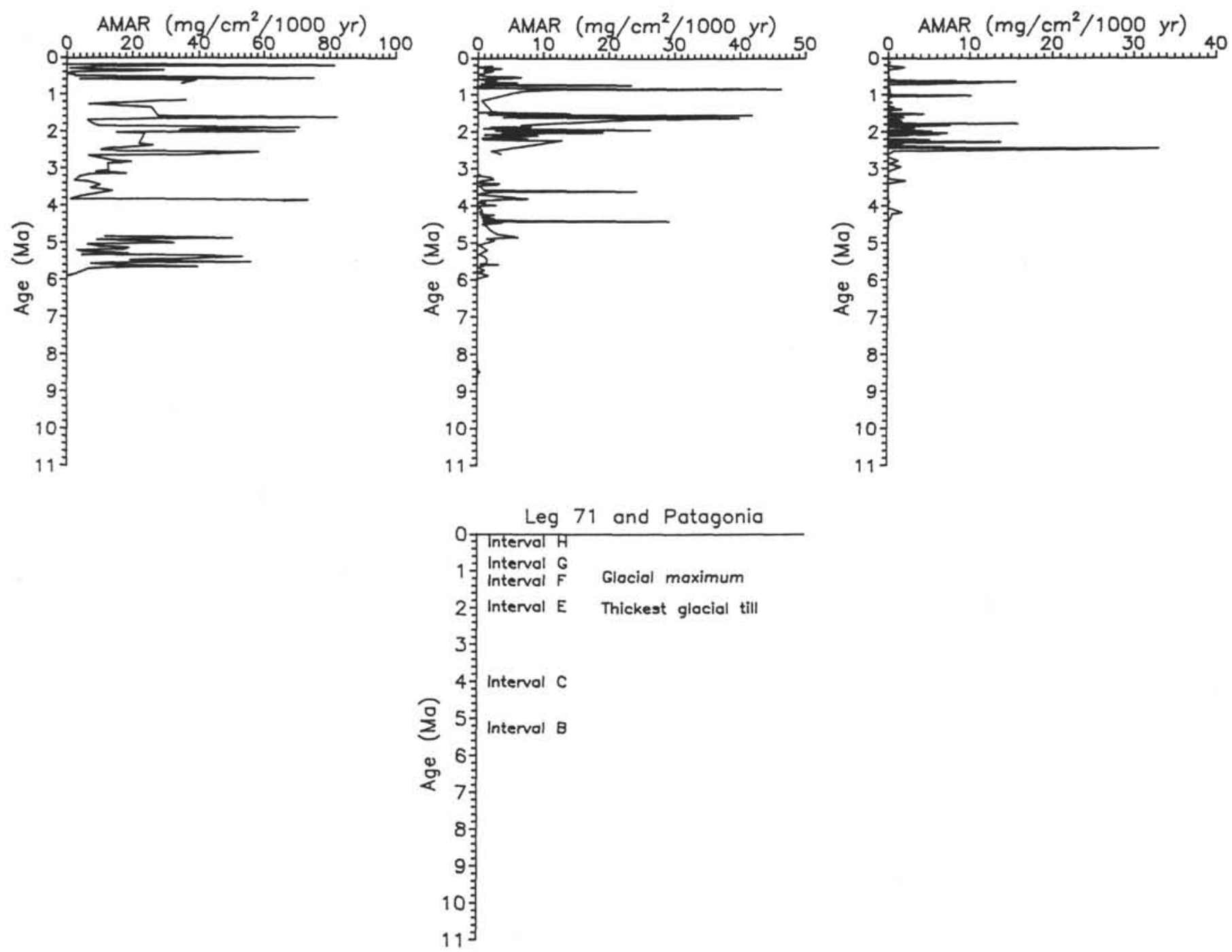

Figure 5. Comparison of ice-rafted debris apparent mass accumulation rates during the past $11.0 \mathrm{Ma}$ among Sites 699 , 701, and 704, Leg 71 ice-rafting intervals, and major glacial events in Patagonia. 